ARTICLE

DOI: $10.1038 / s 41467-018-06629-9$

\title{
Exceptional catalytic effects of black phosphorus quantum dots in shuttling-free lithium sulfur batteries
}

\author{
Zheng-Long $\mathrm{Xu}^{1,2}$, Shenghuang Lin' ${ }^{1}$ Nicolas Onofrio ${ }^{1}$, Limin Zhou ${ }^{3}$, Fangyi Shi ${ }^{3}$, Wei Lu' ${ }^{1}$ Kisuk Kang ${ }^{2}$, \\ Qiang Zhang (i) ${ }^{4} \&$ Shu Ping Lau (1) ${ }^{1}$
}

Lithium sulfur batteries with high energy densities are promising next-generation energy storage systems. However, shuttling and sluggish conversion of polysulfides to solid lithium sulfides limit the full utilization of active materials. Physical/chemical confinement is useful for anchoring polysulfides, but not effective for utilizing the blocked intermediates. Here, we employ black phosphorus quantum dots as electrocatalysts to overcome these issues. Both the experimental and theoretical results reveal that black phosphorus quantum dots effectively adsorb and catalyze polysulfide conversion. The activity is attributed to the numerous catalytically active sites on the edges of the quantum dots. In the presence of a small amount of black phosphorus quantum dots, the porous carbon/sulfur cathodes exhibit rapid reaction kinetics and no shuttling of polysulfides, enabling a low capacity fading rate $(0.027 \%$ per cycle over 1000 cycles) and high areal capacities. Our findings demonstrate application of a metal-free quantum dot catalyst for high energy rechargeable batteries.

\footnotetext{
${ }^{1}$ Department of Applied Physics, The Hong Kong Polytechnic University, Hong Kong, China. ${ }^{2}$ Department of Materials Science and Engineering, Research Institute of Advanced Materials (RIAM), and Center for Nanoparticle Research at Institute of Basic Science (IBS), Seoul National University, Seoul 08826, Republic of Korea. ${ }^{3}$ Department of Mechanical Engineering, The Hong Kong Polytechnic University, Hong Kong, China. ${ }^{4}$ Beijing Key Laboratory of Green Chemical Reaction Engineering and Technology, Department of Chemical Engineering, Tsinghua University, 100084 Beijing, China. These authors contributed equally: Zheng-Long Xu, Shenghuang Lin, Nicolas Onofrio. Correspondence and requests for materials should be addressed to

Q.Z. (email: zhang-qiang@mails.tsinghua.edu.cn) or to S.P.L. (email: apsplau@polyu.edu.hk)
} 
T he development of portable electronic devices drives a search for energy storage systems with lower cost, higher energy density, and better safety than current Li-ion batteries. Among alternative batteries, lithium-sulfur batteries (LSBs) generate interest due to advantages of a high energy density of $2600 \mathrm{~W} \mathrm{~h} \mathrm{~kg}^{-1}$ and the low cost of sulfur feedstock ${ }^{1,2}$. However, the practical application of LSBs has been plagued by several fundamental challenges ${ }^{3-5}$, including the insulating nature of sulfur and lithium sulfides, the large volume expansion of sulfur, and the diffusion of lithium polysulfides (LiPSs) intermediates during charge/discharge cycles. The last obstacle further induces shuttling effects, self-discharge phenomena, and re-distribution of active particles, leading to rapid capacity degradation. Therefore, achieving the confinement of polysulfides in cathodes is a high priority among the primary research of LSBs.

Research over the past decade has brought progress in stabilizing LiPSs by physical and chemical immobilization approaches. Carbon nanomaterials have been systematically designed with high electrical conductivities, desirable pores, and controlled dimensions to host sulfur particles to physically confine $\mathrm{LiPSs}^{6-9}$. Unfortunately, weak interactions between non-polar carbon and polar LiPSs lead to failure in long-term performance of LSBs, causing gradual capacity decay and poor rate performance ${ }^{10}$. Chemical trapping of LiPSs with a polar host ${ }^{11}$, such as N-doped carbon $^{12}$ and metal dichalchogenide ${ }^{13}$, has proven effective for preventing diffusion of LiPSs via polar-polar interactions ${ }^{14}$ or Lewis acid-based bonding. ${ }^{15}$ However, in most cases, chemical immobilization is rather limited for improving battery performance because the blocked LiPSs cannot be effectively reused ${ }^{16,17}$. For example, LiPSs can be strongly adsorbed by $\mathrm{TiO}_{2}$, but full conversion to $\mathrm{Li}_{2} \mathrm{~S}$ is difficult due to the low electrical conductivity of $\mathrm{TiO}_{2}{ }^{18}$. Although polar, conductive chemical traps, such as $\mathrm{CoS}_{2}{ }^{19}$ and $\mathrm{WS}_{2}{ }^{20}$, have been prepared as sulfur hosts, the high gravimetric density of metal-containing compounds and the limited number of adsorption sites discourages high energy density of sulfur cathodes. Therefore, the effective trapping and conversion of LiPSs remain as important challenges toward achieving high-performance LSBs.

Black phosphorus (BP) is the most thermodynamically stable allotrope of phosphorus, with low resistivity ranging between 0.48 and $0.77 \Omega \mathrm{cm}^{21}$ and an exceptionally high room-temperature hole mobility of $\sim 1000 \mathrm{~cm}^{2} \mathrm{~V}^{-1} \mathrm{~s}^{-122}$. BP also possesses a low density of $2.69 \mathrm{~g} \mathrm{~cm}^{-323}$, a good bulk conductivity of $\sim 3 \mathrm{~S} \mathrm{~cm}^{-124}$, a fast Li-ion diffusion constant ${ }^{25}$, and high binding energies with sulfur $^{26}$. These properties suggest that BP can chemically bind with LiPSs and convert them immediately to $\mathrm{Li}_{2} \mathrm{~S}$ through good electrical conductivity and fast Li-ion diffusion, without discernibly compensating the mass fraction of active materials. Fewlayered BP flakes have been adopted in separators ${ }^{26}$ or current collectors $^{27}$ to suppress the diffusion of LiPSs, leading to enhanced cyclic stability of LSBs. However, to trap flooded LSPs by the terrace sites of micro-sized BP flakes, a high loading ( $\approx 1 \mathrm{wt} \%$ with respect to the sulfur weight in electrodes) of BP was employed ${ }^{27}$. Considering the $\mathrm{Li}_{2} \mathrm{~S}_{6}$ catholyte with a theoretical capacity of $1299 \mathrm{~mA} \mathrm{~h} \mathrm{~g}^{-1}$ that was used in that work, the $10 \mathrm{wt} \%$ of electrochemically inert BP may reduce the energy density advantage of LSBs. If edge sites of BP are also effective in trapping LiPSs, the amount of BP additives could be significantly decreased by using nanoscale BP particles, for example, BP quantum dots (BPQDs).

In this contribution, we systematically investigate the catalytic properties of the nanostructured $\mathrm{BP}$ and propose a metal-free $\mathrm{BPQD}$ as a robust catalyst for advanced LSBs. Based on adsorption and electrocatalytic studies, we find that the edge sites of the nanostructured BP are key to the effective immobilization of LiPSs. The edge-selective catalytic property of the BPQDs is further validated using density functional theoretical (DFT) calculations, where the zig-zag (ZZ) terminated BP flakes present stronger binding energies with $\mathrm{Li}_{2} \mathrm{~S}$ at the edges than at terrace sites. These results suggest that adsorptivity of LiPS in BP can be largely increased by downsizing BP flakes to QDs. As a proof of concept, we integrate a small amount of BPQDs (2 $\mathrm{wt} \%$ of the cathode weight) with a sulfur/porous carbon fiber cathode, the LSBs exhibit no diffusion of polysulfides as well as excellent battery performance, including high-rate capability $\left(784 \mathrm{~mA} \mathrm{~h} \mathrm{~g}^{-1}\right.$ at $4 \mathrm{C})$ and exceptional cyclic stability $(0.027 \%$ capacity fade per cycle for 1000 cycles). Finally, an impressive capacity retention of near $90 \%$ is observed for high sulfur loading cathodes (up to 8 $\mathrm{mg} \mathrm{cm}^{-1}$ ) for 200 deep cycles under lean electrolyte conditions.

\section{Results}

Rational design of a black phosphorus catalyst. Twodimensional (2D)-layered crystals usually exhibit strong interatomic covalent bonding and weak interlayer van der Waals interactions, rendering different chemical activities of the atoms at terrace and edge sites. The edge sites tend to possess higher electrochemical catalytic activity than the terrace counterparts in catalysis due to their under-coordinated atomic structures ${ }^{28-31}$. Understanding edge-selective catalytic feature is beneficial in designing catalysts by downsizing the particle size to introduce more active sites. BP is a single-element layered material with one phosphorus atom bonded to three adjacent neighbors to form a honeycomb network, similar to graphite and metal dichalcogenides.

To explore whether BP also possesses such unique edgeselective catalytic properties in LSBs, we rationally prepared different-sized BP flakes through probe sonication and centrifugation of bulk BP crystals in N-methyl-2-pyrrolidone (NMP) solution $^{32,33}$. The as-obtained $\mathrm{BP}$ products were designated as BP-4K, BP-8K according to their centrifugation rates of 4000 and $8000 \mathrm{rpm}$, respectively, and BPQD (centrifuged at 12,000 rpm). It is noted that the concentrations of the three BP solutions were controlled as about $0.55 \mathrm{mg} \mathrm{mL}^{-1}$, as determined by inductive coupled plasma with atomic emission spectroscopy (ICP-AES) ${ }^{23}$.

Figure $1 \mathrm{a}$ and $\mathrm{b}$ shows the transmission electron microscopy (TEM) images of the BP- $4 \mathrm{~K}$ and BP-8K flakes, which reveal sizes of about 800 and $300 \mathrm{~nm}$, respectively, indicating that smaller flakes were obtained at higher centrifugation rate. The structural features of the BP flakes were investigated by high-resolution TEM (HRTEM) and the selected area electron diffraction patterns (SAED). As shown in Fig. 1c, the lattice constant of $0.21 \mathrm{~nm}$ is indexed to (002) plane of BP crystal ${ }^{34}$, consistent with the sharp diffraction spots in the inset. The TEM image of the BPQD in Fig. 1d shows an impressive monodispersion of the BPQDs. The QD size is approximatively $4.5 \mathrm{~nm}$ with lattice fringes of 0.33 and $0.21 \mathrm{~nm}$ (Fig. 1e, f), assigned to (021) and (002) planes of BP crystal $^{32,33}$, respectively. The dimensions of the three BP samples were also characterized with atomic force microscope (AFM). Supplementary Fig. 1 shows the BP-4K, BP-8K, and BPQD with thickness $\times$ particle size of about $6.1 \times 800 \mathrm{~nm}, 4.3 \times 300 \mathrm{~nm}$, and $2.5 \times 4.5 \mathrm{~nm}$, respectively. Based on the dimensions of differentsized BP flakes, we can roughly estimate the exposed edge density by $S_{\text {edge }} / S_{\text {whole }}$, where $S_{\text {edge }}$ and $S_{\text {whole }}$ refer to the area of exposed edge and the whole surface, respectively. For simplicity, we set the flake as a rectangle prism with two equal edge lengths and QD as a cylinder. The BP-4K, BP-8K and BPQD present $S_{\text {edge }} / S_{\text {whole }}$ of $1.5 \%, 2.8 \%$, and $52.6 \%$, respectively, implying the highest exposed edge density of QD structure ${ }^{35}$. The chemical structure of the BPQDs was further probed by Raman spectroscopy. Figure $1 \mathrm{~g}$ shows three featured Raman peaks at $360.8,438.1$, and $466.2 \mathrm{~cm}^{-1}$, referring to $\mathrm{A}_{\mathrm{g}}{ }^{1}, \mathrm{~B}_{2 \mathrm{~g}}$, and $\mathrm{A}_{\mathrm{g}}{ }^{2}$ modes, respectively. Compared to 

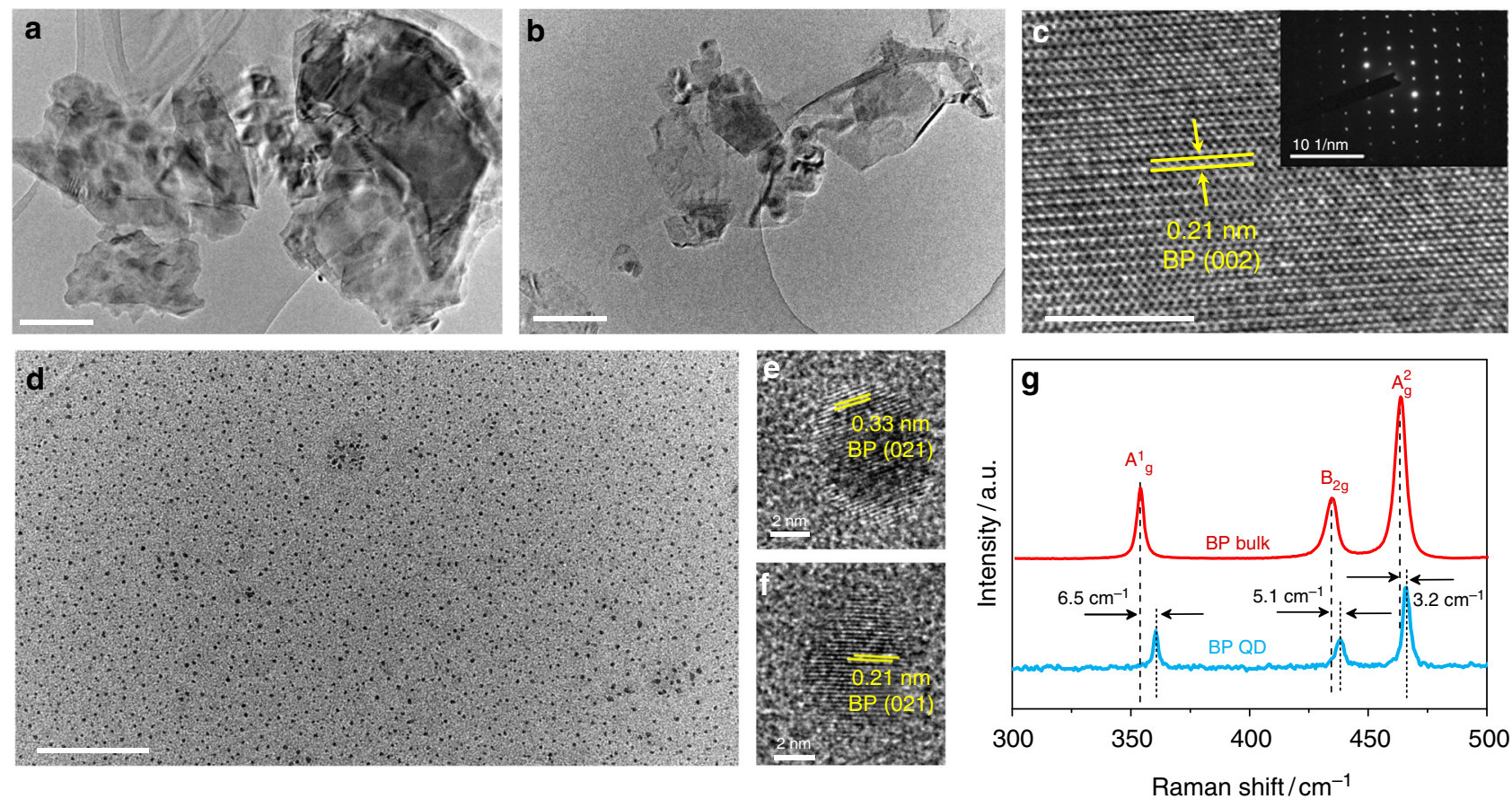

Fig. 1 Morphological and structural characterization of the black phosphorus flakes. a Transmission electron microscopy (TEM) image of black phosphorus (BP) that was centrifuged at $4000 \mathrm{rpm}$ (BP-4 K), b TEM image of BP centrifuged at $8000 \mathrm{rpm}$ (BP-8K), c high-resolution TEM (HRTEM) image and corresponding selected area electron diffraction (SAED) pattern (inset) of BP-8K, d-f TEM and HRTEM images of black phosphorus quantum dots (BPQDs) with monodispersion and high crystallinity, $\mathbf{g}$ Raman spectra of bulk BP (red) and BPQD (blue) on a silicon (Si) substrate. Scale bars, $200 \mathrm{~nm}$ $(\mathbf{a}, \mathbf{b}) ; 5 \mathrm{~nm}(\mathbf{c}) ; 10 \mathrm{~nm}^{-1}$ inset (c); $100 \mathrm{~nm}(\mathbf{d}) ; 2 \mathrm{~nm}(\mathbf{e}, \mathbf{f})$

the bulk BP, the three modes of the BPQD are red-shifted by around $6.5,5.1$, and $3.2 \mathrm{~cm}^{-1}$, respectively, probably due to the thin thickness and small lateral dimensions of the QDs ${ }^{23,32,33}$.

Exploration of catalytic effects of black phosphorus flakes. The catalytic effects of $\mathrm{BP}$ flakes on the polysulfide redox reaction were investigated by potentiostatic discharge deposition of $\mathrm{Li}_{2} \mathrm{~S}$ from $\mathrm{Li}_{2} \mathrm{~S}_{8}$ tetraglyme solution on different substrates, including carbon fiber $(\mathrm{CF})^{35}, \mathrm{CF} / \mathrm{BP}-4 \mathrm{~K}, \mathrm{CF} / \mathrm{BP}-8 \mathrm{~K}$, and CF/BPQD. The amounts of the BP flakes dispersed on $\mathrm{CF}$ substrates were controlled to be the same. Galvanostatic discharge was conducted to $2.06 \mathrm{~V}$ to consume most long-chain polysulfides before applying a $0.01 \mathrm{~V}$ overpotential to drive the formation of $\mathrm{Li}_{2} \mathrm{~S}^{18,36-38}$. Figure $2 \mathrm{a}$ exhibits the potentiostatic discharge curves of the aforementioned electrodes at $2.05 \mathrm{~V}$. Fits of the capacity contributions of the polysulfide reduction and the deposition of $\mathrm{Li}_{2} \mathrm{~S}$ are represented by dark and light colors, respectively (See detailed information in Supplementary Fig. 2). The capacities of the $\mathrm{Li}_{2} \mathrm{~S}$ precipitation on $\mathrm{CF}, \mathrm{CF} / \mathrm{BP}-4 \mathrm{~K}, \mathrm{CF} / \mathrm{BP}-8 \mathrm{~K}$, and $\mathrm{CF} / \mathrm{BPQD}$ are determined to be $37.5,62.7,82.2$, and $174.6 \mathrm{~mA} \mathrm{~h} \mathrm{~g}^{-1}$, respectively, based on the sulfur weight in catholyte. It manifests that the $\mathrm{Li}_{2} \mathrm{~S}$ deposition capacity increases when decreasing BP flake size.

The morphology of the precipitated $\mathrm{Li}_{2} \mathrm{~S}$ on different substrates was observed by scanning electron microscopy (SEM). As shown in Fig. $2 b$, the $\mathrm{Li}_{2} \mathrm{~S}$ on CF surface is a discrete coating, suggesting insufficient deposition of the $\mathrm{Li}_{2} \mathrm{~S}$ due to the poor affinity between polysulfides and non-polar carbon. This deposition type is ascribed to a very high energy barrier for the redox reaction of polysulfides on $\mathrm{CF}^{36,38}$. The amounts of the $\mathrm{Li}_{2} \mathrm{~S}$ deposited on the $\mathrm{CF} / \mathrm{BP}-4 \mathrm{~K}$ and $\mathrm{CF} / \mathrm{BP}-8 \mathrm{~K}$ are significantly improved with a uniform deposition. The enhanced precipitation of the $\mathrm{Li}_{2} \mathrm{~S}$ can be attributed to the strong interactions between polysulfides and the conductive polar $\mathrm{BP}$ flakes $^{26,27}$, thus lowering the precipitation energy barriers and increasing nucleation sites. Interestingly, we observe radial growth of three-dimensional (3D) $\mathrm{Li}_{2} \mathrm{~S}$ particles on the CF/BPQD, in agreement with the highest $\mathrm{Li}_{2} \mathrm{~S}$ precipitation capacity depicted in Fig. 2a. In general, the precipitation of the $\mathrm{Li}_{2} \mathrm{~S}$ on carbon substrate follows a $2 \mathrm{D}$ lateral growth theory along the polysulfide- $\mathrm{Li}_{2} \mathrm{~S}$-substrate tri-phase boundary ${ }^{37}$. The $3 \mathrm{D}$ growth of $\mathrm{Li}_{2} \mathrm{~S}$ was only observed in LSBs with TiC catalyst ${ }^{36}$ and an organic redox mediator ${ }^{38}$, which promoted nucleation/ growth of $\mathrm{Li}_{2} \mathrm{~S}$ along the radial direction by regulating the impingement of insulating $\mathrm{Li}_{2} \mathrm{~S}$. Analogically, the BPQDs facilitate $3 \mathrm{D}$ precipitation of the $\mathrm{Li}_{2} \mathrm{~S}$ by the strong adsorption and rapid conversion of LiPSs on the numerous catalytically active sites. Compared to the routine $2 \mathrm{D}$ deposition of $\mathrm{Li}_{2} \mathrm{~S}$, such anomalous $3 \mathrm{D}$ structure of $\mathrm{Li}_{2} \mathrm{~S}$ enables higher usage of LiPSs in cathodes, thus more effectively suppressing the diffusion of LiPSs to bulk electrolyte. The above results suggest the effective catalytic effect of the BP flakes in LiPS conversion reaction and that BPQDs outperform the larger BP flakes.

In addition, the catalytic effect of BPQDs was further verified via linear sweep voltammetry (LSV) and symmetric cyclic voltammetry $(\mathrm{CV})$ measurements of the $\mathrm{CF}$ and CF/BPQD electrodes in the presence of $\mathrm{Li}_{2} \mathrm{~S}_{4}$ catholyte (Supplementary Fig. 3) $30,39,40$. The CF/BPQD electrodes exhibited higher reaction peaks in LSV curves and a larger exchange current density of 98 $\mu \mathrm{A} \mathrm{cm}^{-2}$ than $75 \mu \mathrm{A} \mathrm{cm}^{-2}$ for CF calculated from Tafel plots. These results further evidence that the kinetics of polysulfide redox reactions are effectively improved by BPQDs.

To disclose the reason why BPQDs exhibited superior catalytic property, we examined the interactions between LiPSs and BP flakes through X-ray photoelectron spectroscopy (XPS) and ultraviolet-visible (UV-Vis) absorption measurements. The nature of the interactions between LiPSs and BPQDs was probed by XPS as shown in Fig. $2 c$. In the deconvoluted P $2 p$ spectra, 
a
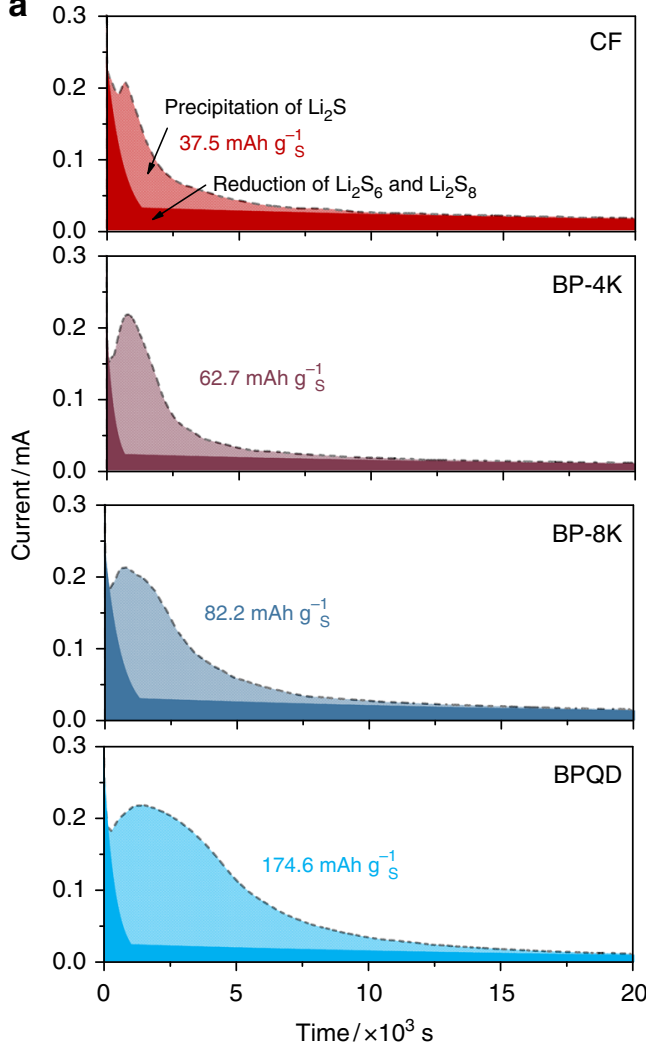

b
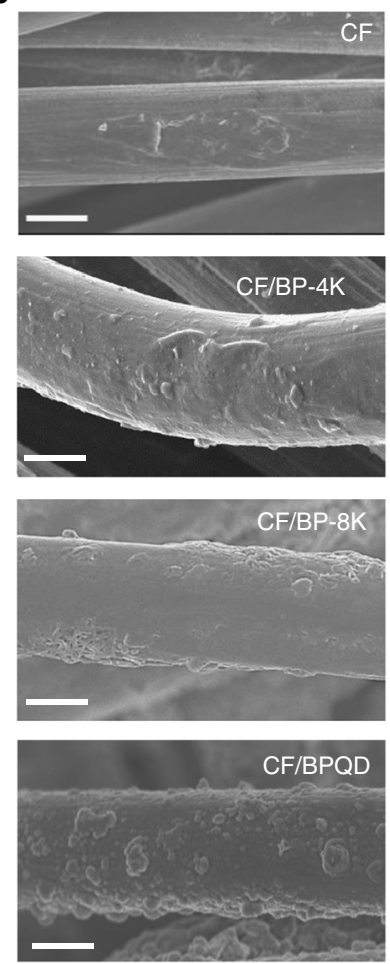

C
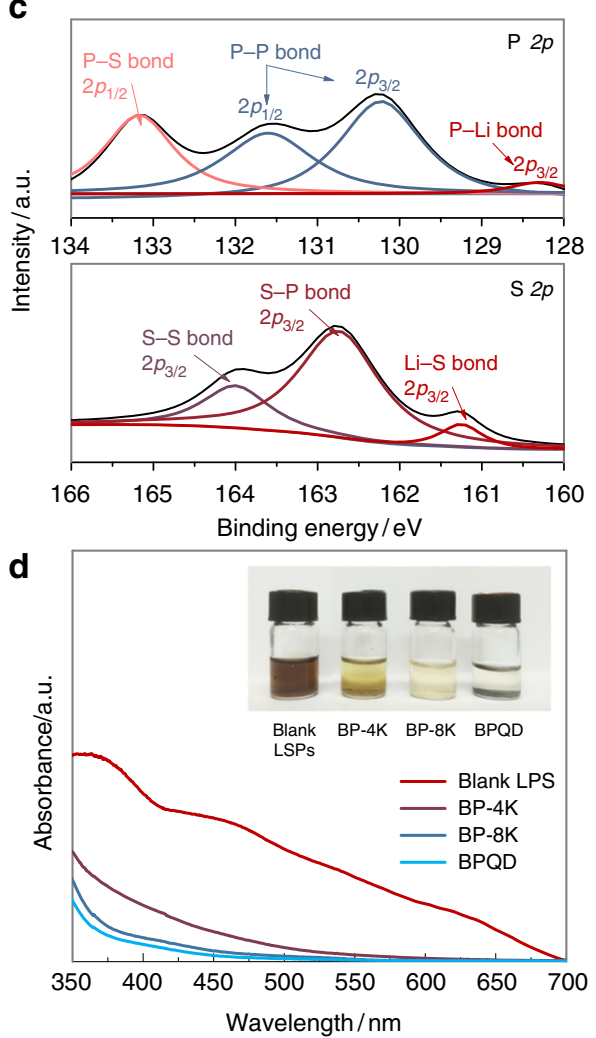

Fig. 2 Black phosphorus flakes for lithium polysufide conversion and adsorption. a Potentiostatic discharge curves of $\mathrm{Li}_{2} \mathrm{~S}_{8}$ tetraglyme solution on different substrates at $2.05 \mathrm{~V}$. The dark/light colors indicate the reduction of $\mathrm{Li}_{2} \mathrm{~S}_{8} / \mathrm{Li}_{2} \mathrm{~S}_{6}$ and the precipitation of $\mathrm{Li}_{2} \mathrm{~S}$, respectively. $\mathbf{b}$ scanning electron microscopy (SEM) images showing the precipitation of $\mathrm{Li}_{2} \mathrm{~S}$ on different substrates as indicated in $\mathbf{a}, \mathbf{c} X$-ray photoelectron spectroscopy (XPS) spectra for $\mathrm{P} 2 p$ and $\mathrm{S}$ $2 p$ of the black phosphorus (BP) flakes adsorbed with lithium polysulfides (LiPSs), $\mathbf{d}$ ultraviolet-visible (UV-vis) spectra of LiPS with variation in color upon adsorption by different-sized BP flakes. Scale bar, $5 \mu \mathrm{m}$ (b). (CF is carbon fiber, BP-4K is BP that was centrifuged at $4000 \mathrm{rpm}$, BP-8K was centrifuged at 8000 rpm, BPQD is black phosphorus quantum dot)

apart from the two $\mathrm{P}-\mathrm{P}$ bonds at $130.1 \mathrm{eV}$ and $131.6 \mathrm{eV}$ referring to $\mathrm{P} 2 p_{3 / 2}$ and $\mathrm{P} 2 p_{2 / 1}$ signals in pristine BPQD (Supplementary Fig. 4) $)^{26}$, two additional peaks at $128.3 \mathrm{eV}$ and $133.0 \mathrm{eV}$, corresponding to P-Li $2 p_{3 / 2}$ and P-S $2 p_{2 / 1}$, are detected. Moreover, a strong S-P $2 p_{3 / 2}$ bond at $162.7 \mathrm{eV}$ is observed in $\mathrm{S} 2 p$ spectrum. These results indicate that the BPQDs strongly interact with LiPSs via both P-S and P-Li bonds, resulting in high adsorptivity between the materials. Static adsorption of the LiPSs was conducted by adding same amounts (about $1 \mathrm{mg}$ ) of BP flakes separately in $10 \mathrm{~mL} \mathrm{Li} \mathrm{L}_{8}$ tetraglyme solution, which showed distinct bulk adsorptivity with a sequence of BPQD $>\mathrm{BP}-8 \mathrm{~K}>$ BP-4K (inset Fig. 2d), consistent with the UV-Vis spectra results. The different LiPS adsorptivities are related to the population of the active sites, suggesting the most adsorbing sites on the BPQDs among the three BP samples. Considering that the difference of terrace areas for the $\mathrm{BP}$ flakes with the same mass is relatively trivial, the dramatically increased number of edge sites on the QDs are suggested to contribute to the significant difference in adsorptivity.

To quantify the relative adsorptivity between terrace and edge sites of the BPQDs with LiPS, we performed DFT calculations. Figure 3 a shows snapshots of the atomic structures of $\mathrm{Li}_{2} \mathrm{~S}_{n}(n=$ 1-4) adsorbed on the terrace sites of free-standing BP monolayer as well as adsorbed on edge sites of bilayers BP nanoribbons. Figure $3 \mathrm{~b}$ presents the binding energies, giving rise to two interesting results: (i) the binding energy of $\mathrm{Li}_{2} \mathrm{~S}_{n}$-BP decreases when the size of the adsorbed molecule increases ${ }^{26,27}$; (ii) the binding energies of $\mathrm{Li}_{2} \mathrm{~S}_{n}$ adsorbed at the edge of $\mathrm{BP}$ nanoribbons are significantly larger than those at terrace sites. Binding energies computed with and without van der Waals (vdW) correction indicate that the vdW contribution to bonding (i.e., the binding energy without vdW is divided by the binding energy with vdW) is always smaller when the LiPS is adsorbed at edge sites (see inset of Fig. 3b). Bader charge analysis ${ }^{41}$ shows that in average, partial charges on $\mathrm{Li}$ atoms are similar whether the LiPS is adsorbed at terrace or edge sites, equaling to $-0.86 \mathrm{eV}$. However, we found lower partial charges on S-atom part of LiPS adsorbed at edge sites, suggesting less ionic character of the bond. The stronger covalent character of bonds formed between BP edges and LiPS can be attributed to the high reactivity of the under-coordinated $\mathrm{P}$-atoms at the zig-zag terminated edges compared to stoichiometric $\mathrm{P}$-atoms at the terrace ${ }^{42,43}$. In addition, the exposed edge area of BP flakes can be rationally increased by downsizing BP. For example, when a $\mathrm{BP}$ rectangle prism with two equal edge lengths of $200 \mathrm{~nm}$ and a thickness of $3 \mathrm{~nm}$ is divided into BPQDs with the same thickness and equal edge lengths of $4 \mathrm{~nm}$, the exposed edge surface area increases by $\sim 25$ times (Fig. 3c), in agreement with the improved $S_{\text {edge }} / S_{\text {whole }}$ ratio from $\mathrm{BP}-8 \mathrm{~K}$ to BPQD. At the same time, the number of particles increases from 1 to 2500 , with largely decreased particle size, ensuring uniform dispersion of catalysts within host materials. Together with the above experimental results, we can conclude that BP flakes exhibit an edge-selective catalytic property. Further, a QD structure with a high aspect ratio and numerous edge sites should be an ideal choice to improve LiPS adsorptivity and redox reaction kinetics in LSBs. 
a

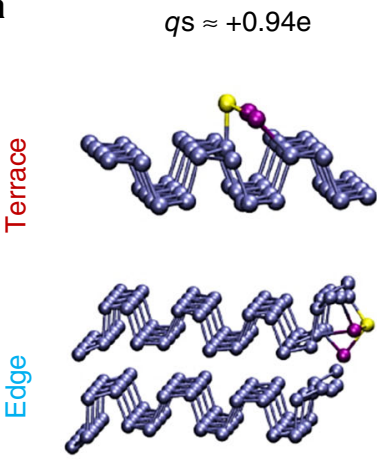

$q s \approx+0.93 e$ $q s \approx+0.87 e$


$q \mathrm{~s} \approx+0.52 \mathrm{e}$


$q s \approx+0.37 e$
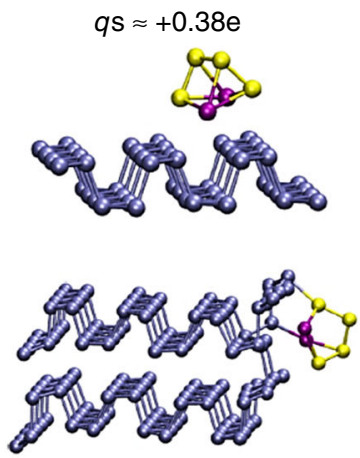

$q \mathrm{~s} \approx+0.33 e$
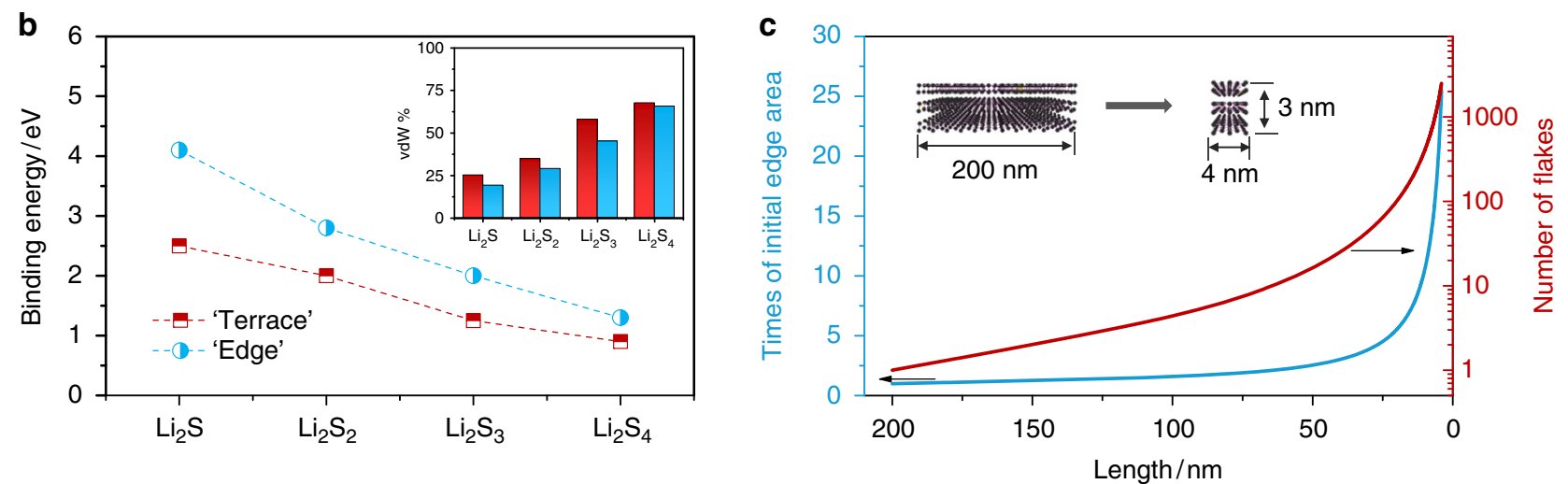

Fig. 3 Calculation of lithium polysulfide adsorption on black phosphorus flakes. a Snapshots of lithium polysulfides (LiPSs) adsorbed on terrace and edge sites of black phosphorus (BP) monolayer and nanoribbons, respectively. The partial charge on the sulfur atom is defined as qs, $\mathbf{b}$ binding energies of LiPSs adsorbed on terrace and edge sites of BP, the inset shows the fraction of van der Waals (vdW) contribution to the bond, $\mathbf{c}$ the increase of exposed edge area and the number of flakes by downsizing a large BP flake to black phosphorus quantum dots (BPQDs)

Integrate black phosphorus quantum dots with sulfur/carbon cathodes. To apply the BPQD catalyst in actual working cathodes, we integrated the BPQDs and sulfur particles with porous carbon nanofibers (PCNFs). The PCNFs are consist of numerous hollow graphitic carbon spheres with open pores (See Methods and Supplementary Fig. 5), allowing the impregnation of sulfur particles and BPQDs inside. ${ }^{44}$ It is noted that the PCNF host possesses a high pore volume of $0.82 \mathrm{~cm}^{3} \mathrm{~g}^{-1}$ and a large surface area of $1277 \mathrm{~m}^{2} \mathrm{~g}^{-1}$, both of which are important in delivering high sulfur loading. Figure $4 \mathrm{a}$ and $\mathrm{b}$ shows TEM images of the PCNF and PCNF/S/BPQD, respectively. Obviously, the hollow carbon spheres in the PCNF are filled with sulfur particles. The HRTEM image of the PCNF/S/BPQD in Fig. 4c presents uniformly dispersed BPQD crystals with a fringe space of $0.25 \mathrm{~nm}$ as well as graphitic carbon layers with a lattice range of $0.34 \mathrm{~nm}$. The scanning TEM (STEM) image and energy dispersive spectroscopy (EDS) elemental mapping of an individual fiber presented in Fig. 4d shows that (i) sulfur particles are uniformly integrated with the PCNF host and (ii) BPQDs are homogeneously dispersed among sulfur particles. It is noted that nitrogen doping is also observed in the PCNF/S/BPQD composite, which is derived from the polyacrylonitrile polymer precursor ${ }^{45,46}$. The chemical components of the ternary hybrid material were determined by thermal gravimetric analysis (TGA) to be about $68 \mathrm{wt} \%$ for sulfur and $2 \mathrm{wt} \%$ for BPQDs as shown in Supplementary Fig. 6. PCNF/S composites were also prepared for control experiments.

Electrochemical behaviors of black phosphorus quantum dots/ sulfur/carbon cathodes. The volume expansion of sulfur particles after being fully lithiated to $\mathrm{Li}_{2} \mathrm{~S}$ is theoretically calculated to be $80 \%$. This large volume variation would squeeze lithiated product out of porous carbon host ${ }^{47}$, aggravating the polysulfide diffusion and the capacity degradation of sulfur cathodes. To identify whether the BPQDs can suppress polysulfide diffusion even under large volume expansion of sulfur we conducted a series of in situ experiments, including TEM, transmission battery (TB) and electrochemical impedance spectroscopy (EIS) measurements. In situ TEM investigation was performed by applying an external voltage of $-2 \mathrm{~V}$ to the PCNF/S/BPQD working electrode versus $\mathrm{Li} / \mathrm{Li}_{2} \mathrm{O}$ counter electrode. The gradual structural evolution due to the lithiation of the PCNF/S/BPQD fiber is recorded in Supplementary Movie 1, from which the captured images at different reaction stages are shown in Fig. $4 \mathrm{e}-\mathrm{g}$. It is worth noting that the lithiation process is uniform over the circular cross-section from the contact interface to the PCNF/S/BPQD fiber direction. The SAED patterns indicate that amorphous sulfur particles (Fig. 4h) are fully converted to crystalline $\mathrm{Li}_{2} \mathrm{~S}$ (Fig. 4i) after lithiation ${ }^{48,49}$. To measure the volume expansion, an $817 \mathrm{~nm}$ long fiber with a diameter of 358 $\mathrm{nm}$ was selected, which expanded to $821 \mathrm{~nm}$ in length and 447 $\mathrm{nm}$ in diameter after full lithiation, corresponding to $56.7 \%$ volume expansion. Although this value is lower than the theoretical $80 \%$, HRTEM image shows that the lithiated product spilled over the carbon spheres due to the volume expansion (Supplementary Fig. 7). In real batteries, a 56.7\% volume expansion would induce overflowing of polysulfides and shuttling effect. As a direct evidence, the in situ TB experiments show that the colorless electrolyte around the PCNF/S changed to yellow due to overflowing of polysulfides during discharging (Supplementary Fig. 8), in sharp contrast to the unchanged color of electrolyte in the PCNF/S/BPQD battery, suggesting effective immobilization of LiPSs by BPQDs. 

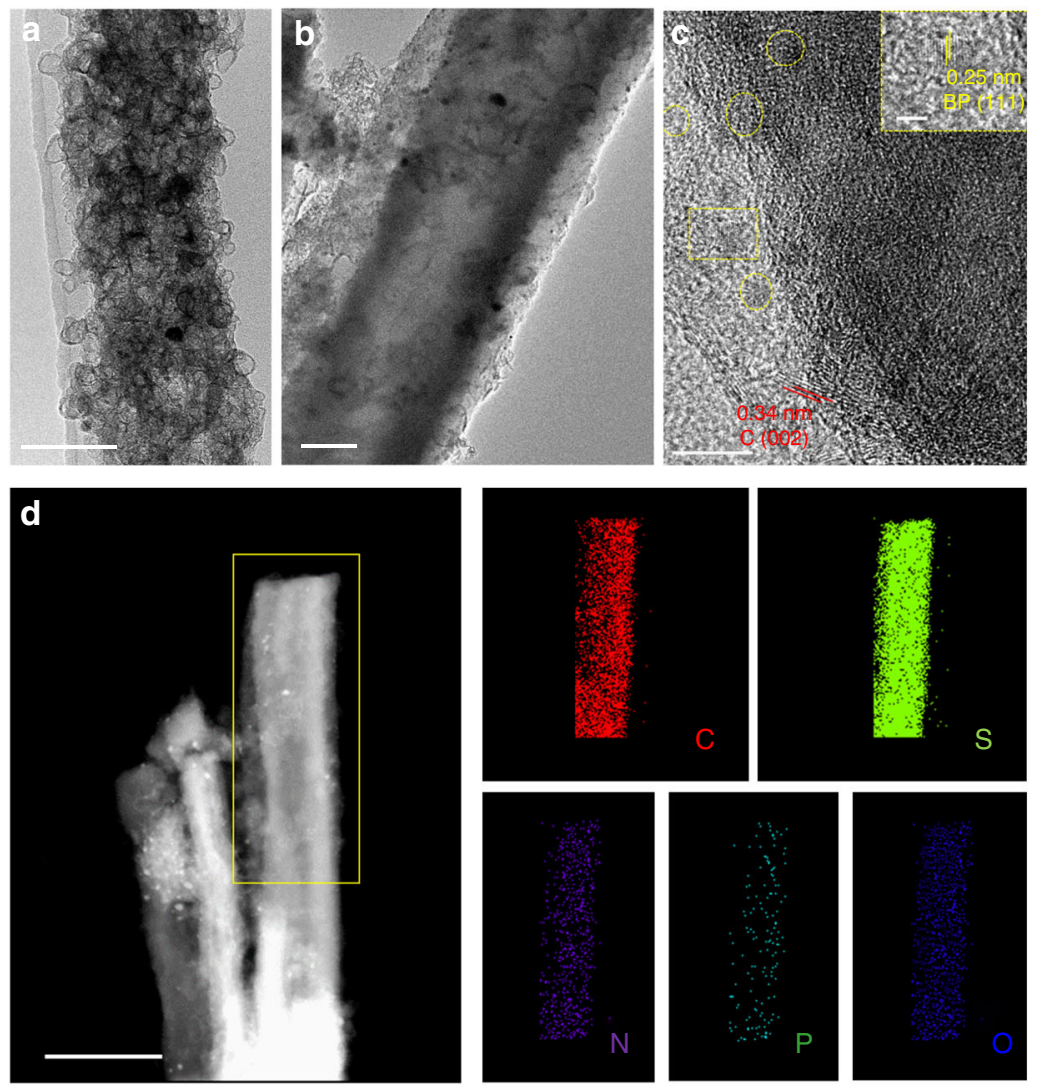
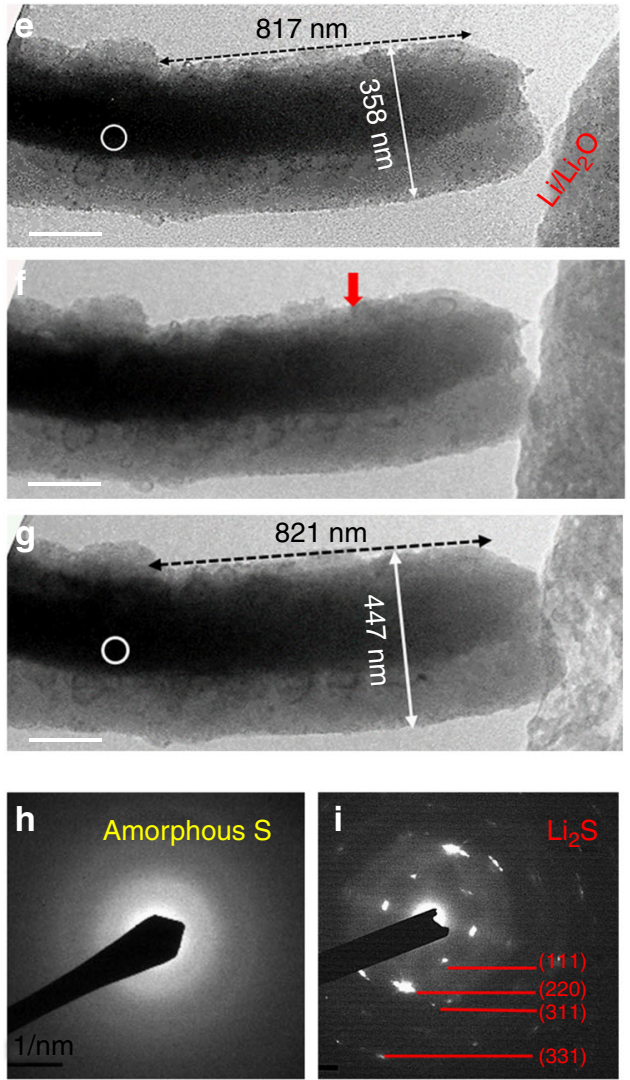

Fig. 4 Integrate black phosphorus quantum dots with porous carbon/sulfur cathodes. a Transmission electron microscopy (TEM) image of the porous carbon nanofiber (PCNF) consisting of numerous hollow carbon spheres, $\mathbf{b}$ TEM image of the porous carbon host in a filled with sulfur particles and black phosphorus quantum dot (BPQD), designated as PCNF/S/BPQD, c high-resolution TEM (HRTEM) image showing the graphitic carbon layers of the PCNF and the uniform dispersion of BPQD within PCNF/S/BPQD, d scanning tunneling electron microscopy (STEM) image and energy dispersive spectroscopy (EDS) elemental mapping of the PCNF/S/BPQD fibers, e-g TEM images captured during lithiation of a PCNF/S/BPQD fiber, the red arrow in $\mathbf{f}$ refers to the reaction front, $\mathbf{h}$, i selected area electron diffraction (SAED) patterns of the selected area with white circles in $\mathbf{e}$ and $\mathbf{g}$, respectively. Scale bars, $100 \mathrm{~nm}$ (a); $200 \mathrm{~nm}$ (b); $10 \mathrm{~nm}$ (c); $2 \mathrm{~nm}$ inset (c); $500 \mathrm{~nm}$ (d); $200 \mathrm{~nm}(\mathbf{e}-\mathbf{g})$

In situ EIS is a powerful technique to probe the electrochemical impedance of LSBs at different reaction stages ${ }^{50,51}$. Nyquist plots of the PCNF/S/BPQD and the PCNF/S cathodes were collected during the initial lithiation process. The system resistance $\left(R_{\mathrm{s}}\right)$, electrolyte/electrode interface resistance $\left(R_{\text {suf }}\right)$, and charge transfer resistance $\left(R_{\mathrm{ct}}\right)$ obtained from equivalent circuit are plotted in Supplementary Fig. 9. It is noteworthy that $R_{\mathrm{ct}}$ of the $\mathrm{PCNF} / \mathrm{S}$ is greatly increased as compared to that of the PCNF/S/ BPQD, implying diffusion of the LiPSs and shuttling effect of the PCNF/S cathode. For the PCNF/S/BPQD electrode, both $R_{\text {suf }}$ and $R_{\mathrm{ct}}$ remained stable throughout the whole discharge process, indicating that the BPQDs can suppress the outward diffusion of the LiPSs and maintain structural integrity. These findings offer solid evidences for the effective immobilization of the LiPSs by the BPQDs in porous carbon/sulfur electrodes.

The electrochemical performance of the PCNF/S/BPQD electrodes were evaluated between 1.7 and $2.8 \mathrm{~V}$ vs. $\mathrm{Li}^{+} / \mathrm{Li}$ using lithium metal as counter electrode; for comparison, the PCNF/S electrodes were also tested. It is noted that the lithiation of the BP occurs below $1.2 \mathrm{~V}$ vs. $\mathrm{Li}^{+} / \mathrm{Li}^{52}$, thus the BPQDs will not participate in any reaction in the $1.7-2.8 \mathrm{~V}$ voltage range. Figure 5a presents the initial discharge/charge profiles of the $\mathrm{PCNF} / \mathrm{S}$ and PCNF/S/BPQD electrodes at $0.1 \mathrm{C}(1 \mathrm{C}=1675 \mathrm{~mA}$ $\mathrm{g}^{-1}$ ). The two discharge plateaus at $\sim 2.4$ and $2.1 \mathrm{~V}$ are associated with the reduction of sulfur particles to long-chain polysulfides and the subsequent conversion of soluble polysulfides to insoluble lithium sulfides, respectively ${ }^{44-46}$. The reduction of the lithium sulfides to sulfur is presented by the plateau at $2.3-2.4 \mathrm{~V}$ in the corresponding charge curve. Figure 5 a evidently shows that (i) the reversible specific capacity from the PCNF/S/BPQD electrode is much larger than that of the PCNF/S (1234 vs. $907 \mathrm{~mA} \mathrm{~h} \mathrm{~g}^{-1}$, respectively); (ii) the $\mathrm{PCNF} / \mathrm{S} / \mathrm{BPQD}$ electrode presents a much lower polarization between discharge and charge curves than that for the PCNF/S (0.205 vs. $0.278 \mathrm{~V}$, respectively). The higher specific capacity of the PCNF/S/BPQD implies that the sulfur utilization is significantly enhanced by the BPQD via trapping and reusing polysulfides ${ }^{18,27,39}$. The lower polarization suggests higher redox reaction kinetics in the LSB, consistent with the higher exchange current densities mentioned before. In addition, the CV curves of the PCNF/S/BPQD electrodes (Supplementary Fig. 10) show that the low-voltage reduction peak (referring to $\mathrm{Li}_{2} \mathrm{~S}_{4} \rightarrow \mathrm{Li}_{2} \mathrm{~S}$ ) shifts to higher potential and the oxidation peak shifts to lower potential than the PCNF/S, suggesting the catalytic effect of the BPQD on enhancing the oxidation/reduction kinetics of sulfur particles ${ }^{18}$.

Figure 5b shows the cyclic stability of the PCNF/S/BPQD and $\mathrm{PCNF} / \mathrm{S}$ electrodes at a low current density of $0.1 \mathrm{C}$. The PCNF/S/ BPQD exhibited an initial capacity of $1234 \mathrm{mAh} \mathrm{g}^{-1}$ and maintained at $1072 \mathrm{~mA} \mathrm{~h} \mathrm{~g}^{-1}$ after 200 cycles, rendering an ultralow capacity fading rate of $0.06 \%$ per cycle. In contrast, the PCNF/S delivered a climax capacity of $1036 \mathrm{mAh} \mathrm{g}^{-1}$ and maintained at $623 \mathrm{~mA} \mathrm{~h} \mathrm{~g}^{-1}$ after 200 cycles, leading to a highcapacity fading rate of $2 \%$ per cycle. The rapid capacity degradation of the PCNF/S can be evidenced by its lower 

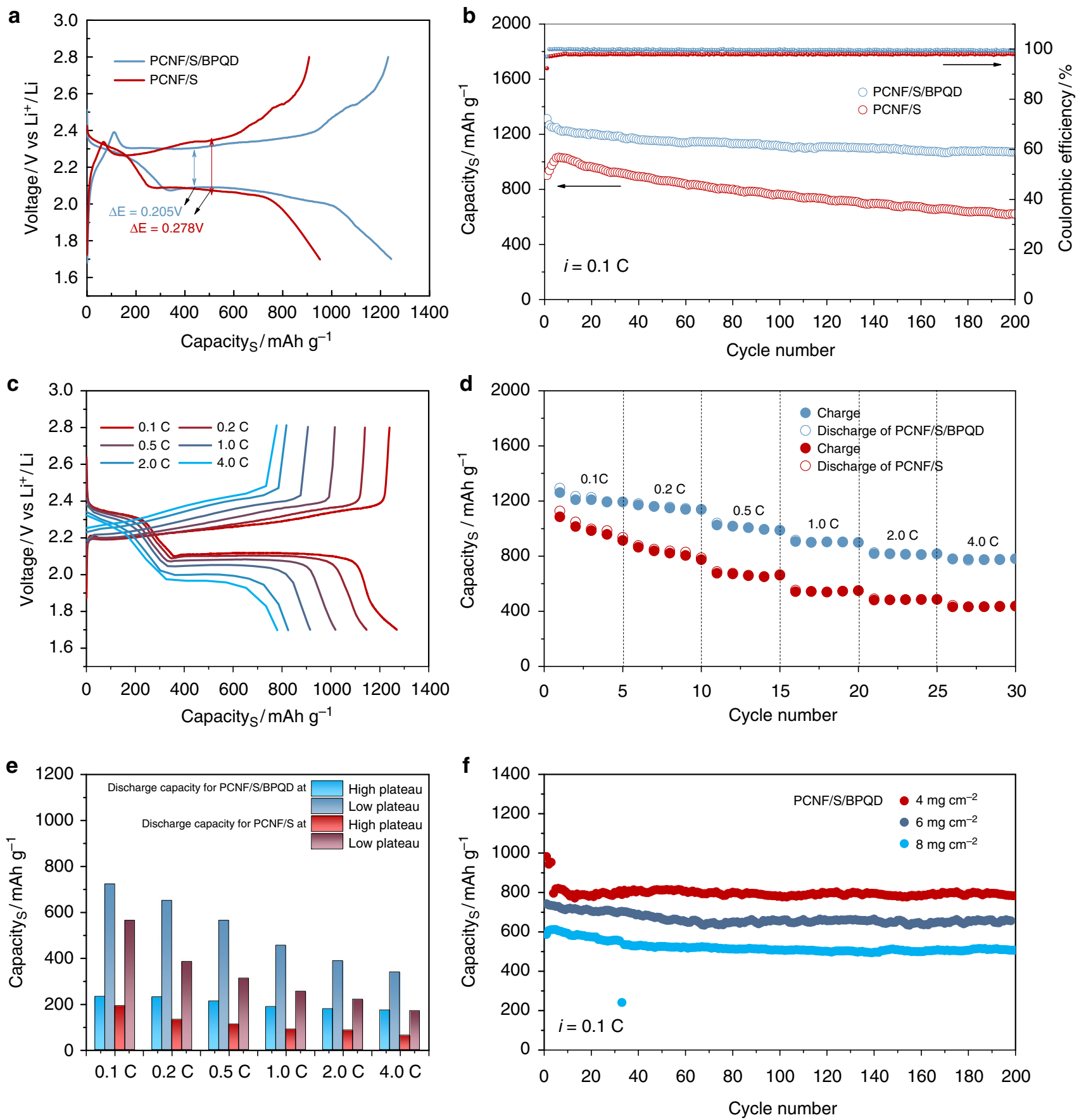

Fig. 5 Electrochemical performance of cathodes. a Initial discharge-charge profiles at $0.1 \mathrm{C}$ between 1.7 and $2.8 \mathrm{~V}$ vs. Li+/ Li, b cyclic capacities and Coulombic efficiencies at $0.1 \mathrm{C}$ for 200 cycles, c discharge-charge voltage profiles of the porous carbon nanofiber/sulfur/black phosphorus quantum dot (PCNF/S/BPQD) cathode at different rates, $\mathbf{d}$ cyclic capacities at different rates, e high plateau and low plateau discharge capacities derived from rate performance, $\mathbf{f}$ cyclic performance of the PCNF/S/BPQD at $0.1 \mathrm{C}$ for 200 cycles with increasing sulfur loadings of 4,6 , and $8 \mathrm{mg} \mathrm{cm}^{-2}$

Coulombic efficiencies during cycles $(\approx 98 \%$ for $\mathrm{PCNF} / \mathrm{S}$ vs. $\approx 99.9 \%$ for $\mathrm{PCNF} / \mathrm{S} / \mathrm{BPQD}$, respectively). The two electrodes were also cycled in $\mathrm{LiNO}_{3}$-free electrolyte (Supplementary Fig. 11). Although the cyclic capacities for PCNF/S/BPQD are slightly lower than in electrolyte with $\mathrm{LiNO}_{3}$ additive, due to the positive effect of $\mathrm{LiNO}_{3}$ in stabilizing $\mathrm{Li}$ metal and suppressing the shuttle effect of LPS $s^{53,54}$, the cyclic performance is still much better than PCNF/S electrodes. Considering the similar sulfur loading and the same carbon host for two electrodes, it is evident that the differences in cyclic capacities are attributed to the different redox reaction kinetics and entrapment of LiPSs by the
BPQDs, enabling high Coulombic efficiencies and high cyclic capacities. To verify the long-term stability of the PCNF/S/BPQD, the batteries were tested at a low current density of $0.5 \mathrm{C}$ for 500 cycles and a high current density of 2 C for 1000 cycles (Supplementary Fig. 12). It is observed that the PCNF/S/BPQD electrodes present a capacity retention of $74 \%$ over 500 cycles at $0.5 \mathrm{C}$, rendering a capacity fading rate of $0.052 \%$, which is much lower than the $0.163 \%$ for PCNF/S. When cycling at $2 \mathrm{C}, \mathrm{PCNF} /$ S/BPQD presents the excellent capacities of $810 \mathrm{~mA} \mathrm{~h} \mathrm{~g}^{-1}$ at $1 \mathrm{st}$ cycle and $589 \mathrm{~mA} \mathrm{~h} \mathrm{~g}^{-1}$ at 1000th cycle, giving rise to an extremely low capacity decay rate of $0.027 \%$ per cycle. 
The catalytic effect of the BPQDs can be more clearly evaluated by cycling batteries at high rates, which require faster charge transfer and higher redox reaction kinetics at the electrode/ electrolyte interface. The PCNF/S/BPQD exhibits reversible capacities of $1266,1172,1030,910,821$, and $784 \mathrm{~mA} \mathrm{~h} \mathrm{~g}^{-1}$ at $0.1,0.2,0.5,1,2$, and $4 \mathrm{C}$, respectively (Fig. 5c). Both the discharge and charge curves overlap well below the current density of $1 \mathrm{C}$, suggesting excellent kinetics and small polarizations of the PCNF/S/BPQD electrodes. The apparent platforms are still maintained even at high rates above $1 \mathrm{C}$, which can be attributed to the firm chemical interactions between LiPSs and BPQD and the facilitated conversion reactions. The decreased capacity and increased polarization for the PCNF/S/BPQD may be ascribed to the high ohmic resistance at higher current densities $^{55,56}$. In contrast, the $\mathrm{PCNF} / \mathrm{S}$ displays much lower capacities (Fig. 5d) under the same rate cycling conditions. To elucidate reasons for the difference, we separated out the high plateau (at about $2.35 \mathrm{~V}$, referring to generation of polysulfides) and the low plateau (at about $2.1 \mathrm{~V}$, referring to nucleation/ growth of lithium sulfides) discharge contributions to the total capacities at different current rates for the PCNF/S/BPQD and PCNF/S electrodes (Fig. $5 \mathrm{c}$ and Supplementary Fig. 10). The high plateau capacity for PCNF/S/BPQD decreases by ca. $25 \%$ (from 232 to $173 \mathrm{~mA} \mathrm{~h}^{-1}$ ) with current density increasing from 0.1 to $4 \mathrm{C}$ (Fig. 5e), which is much lower than ca. $67 \%$ (from 191 to 63 $\mathrm{mA} \mathrm{h} \mathrm{g}^{-1}$ ) for PCNF/S, confirming the strong immobilization of LiPSs by BPQD in the PCNF/S/BPQD electrode. We also find that the low plateau discharge capacity at $4 \mathrm{C}$ for the PCNF/S/ BPQD maintains $47 \%$ of the capacity at $0.1 \mathrm{C}$, greater than the $30 \%$ for the PCNF/S electrodes. The high discharge capacity retention at low plateau indicates the facilitated conversion reactions catalyzed by the BPQD in PCNF/S/BPQD electrodes. In addition, Supplementary Fig. 10 shows the high and low discharge plateau voltages of PCNF/S and PCNF/S/BPQD electrodes at different $\mathrm{C}$-rates. In contrast to the sharp decrease of low discharge plateau voltage for $\mathrm{PCNF} / \mathrm{S}$ at high rate (i.e., from $2.12 \mathrm{~V}$ at $0.1 \mathrm{C}$ to $1.76 \mathrm{~V}$ at $4 \mathrm{C}$ ), the PCNF/S/BPQD system displays much better performance (i.e., from $2.11 \mathrm{~V}$ at $0.1 \mathrm{C}$ to $1.99 \mathrm{~V}$ at $4 \mathrm{C}$ ), indicating the low polarization and fast redox reaction from the catalytic effect of BPQD in $\mathrm{LSBs}^{27}$. As a final piece of evidence, the PCNF/S/BPQD electrodes exhibit cyclic capacity retentions of $95 \%, 90 \%$, and $89 \%$ after 200 cycles at $0.1 \mathrm{C}$ at high areal sulfur loadings of 4,6 , and $8 \mathrm{mg} \mathrm{cm}^{-2}$ (Fig. 5f), respectively. The impressive stability at high sulfur loadings suggests that the BPQDs can effectively immobilize flooded polysulfides of thick electrodes. It is noteworthy that the $89 \%$ capacity retention for PCNF/S/BPQD cycled under an electrolyte/ sulfur (E/S) ratio of $6.5 \mathrm{~mL} \mathrm{~g}^{-1}$ for 200 cycles is competitive to the state-of-the-art LSBs with low E/S ratios ${ }^{10,57}$. It is believed that better performance under lean electrolyte conditions can be achieved by combining our findings of using BPQD catalysts and optimizations in other components of $\mathrm{LSBs}^{58,59}$. Overall, the excellent electrochemical performance of the PCNF/S/BPQD demonstrates that the BPQDs are highly effective in improving LSB performance with sulfur/carbon cathodes.

\section{Discussion}

We have demonstrated the catalytic effect of the BPQDs for efficient trapping and conversion of LiPSs via a suite of experimental and theoretical studies. To identify the superiority of BPQDs to other reported catalysts for LSBs, we have compared the physicochemical properties and their corresponding electrochemical performance in LSBs in Supplementary Table 1 and Table 2. The unique advantages of the BPQD catalyst for LSBs can be summarized as following: (i) the BPQD as a metal-free catalyst holds the lowest gravimetric density among reported catalysts (Supplementary Table 1) to the best of our knowledge, thus for the same weight fraction of catalyst, the active surface area will far exceed that of metals $(\mathrm{Pt}, \mathrm{Ni})^{39,40}$, metal oxide phases $\left(\mathrm{Fe}_{2} \mathrm{O}_{3}, \mathrm{MnO}_{2}\right)^{60,61}$ and metal nitride materials $(\mathrm{VN}, \mathrm{TiN})^{56,62}$ (ii) The BPQDs with unique edge-preferential LiPS immobilization ability offer more active sites than routine $2 \mathrm{D}$ structures. The QD structure with exposed edge sites outperforms bulk flakes based on the amount of active sites, thus leading to better electrochemical performance. For example, both $\mathrm{WS}_{2}$ and $\mathrm{MoS}_{2}$ flakes exhibited an edge-selective catalytic property for LiPSs; however, the reported rate capacities $\left(\sim 400 \mathrm{~mA} \mathrm{~h} \mathrm{~g}^{-1}\right.$ at $2 \mathrm{C}$ for $\mathrm{MoS}_{2} / \mathrm{CNF} / \mathrm{Li}_{2} \mathrm{~S}_{8}$ and $380 \mathrm{~mA} \mathrm{~h} \mathrm{~g}$ at $1 \mathrm{C}$ for $\left.\mathrm{WS}_{2} / \mathrm{Li}_{2} \mathrm{~S}_{6}\right)^{30}$ are much lower than the current BPQD modified cathode $(784 \mathrm{~mA} \mathrm{~h}$ $\mathrm{g}^{-1}$ at $4 \mathrm{C}$ ). (iii) The BPQD with few-layer thickness is expected to possess high electrical conductivity ${ }^{27}$, which is higher than those for metal oxides ${ }^{60,61}$ and metal carbides ${ }^{36}$, thus ensuring rapid conversion kinetics for trapped $\mathrm{LiPS}$ to $\mathrm{Li}_{2} \mathrm{~S}$ particles. In addition, the physical confinement from the PCNF host also contributes to the excellent electrochemical performance. Postcycled analysis reveals that the overall morphology of the PCNF/ S/BPQD composite was almost intact (Supplementary Fig. 13), indicating the robust structural stability of PCNF host. HRTEM images and elemental mappings show that BPQD crystals and sulfur particles are uniformly confined within the fibers, suggesting the chemical and structural integrity of PCNF/S/BPQD during cycling. Consequently, our PCNF/S/BPQD cathodes exhibit one of the best electrochemical performances among reported catalyst-modified cathodes in terms of cyclic capacities, high-rate capability, and areal capacities at high sulfur loadings (Supplementary Table 2). PCNF/S/BPQD electrodes with a high sulfur loading of $8 \mathrm{mg} \mathrm{cm}^{-2}$ and a low E/S ratio of $\sim 6.5 \mathrm{~mL} \mathrm{~g}^{-1}$ delivered an area capacity of $4.4 \mathrm{~mA} \mathrm{~h} \mathrm{~cm}^{-2}$ after 200 cycles, which is higher than the $4.0 \mathrm{~mA} \mathrm{~h} \mathrm{~cm}^{-2}$ for commercial $\mathrm{LiCoO}_{2}$ cathodes $^{57}$. It is worth noting that high sulfur loading and low E/S ratio are recently considered critical challenges for fabricating practical LSBs to outperform commercial LIBs ${ }^{10}$. We believe that incorporating our highly effective BPQD catalyst with highly concentrated polysulfide catholyte would be a promising strategy to mitigate these issues (Supplementary Fig. 14). Moreover, thanks to the high yield of the top-down synthetic procedure ${ }^{27}$ as well as the low content ( $2 \mathrm{wt} \%)$ needed to boost the LSB performance, the large scale application of BPQDs in LSBs is not problematic.

In summary, we demonstrated the effectiveness of the BPQDs as a catalyst for polysulfide immobilization and conversion in LSBs. By downsizing the particle size of 2D BP flakes, we found that the BPQDs exhibited higher LiPS adsorptivity and larger $\mathrm{Li}_{2} \mathrm{~S}$ precipitation capacity than the large BP flakes. DFT calculations revealed that the edge sites of the $2 \mathrm{D} \mathrm{BP}$ materials showed preferential adsorption of polysulfides, inducing more catalytically active sites than bulk BP flakes, thus dictating the superior catalytic performance of the BPQD. To apply the BPQD in actual cathodes, a small amount of the BPQD was integrated with porous carbon/sulfur composite. Time-sequence TEM images showed a large volume expansion and overflowing of reaction products of the PCNF/S/BPQD fiber during lithiation, however, there was neither diffusion of yellow LiPS in a transparent battery nor an increase in battery impedance for the PCNF/S/BPQD electrodes in EIS measurements, thus demonstrating the effectiveness of the BPQD in immobilizing LiPSs. As a result, the PCNF/S/BPQD electrode exhibited impressive electrochemical performance, including a reversible capacity of $1072 \mathrm{~mA} \mathrm{~h} \mathrm{~g}^{-1}$ after 200 deep discharge/charge cycles at $0.1 \mathrm{C}$, a high-rate capacity of $784 \mathrm{~mA} \mathrm{~h} \mathrm{~g}^{-1}$ at $4 \mathrm{C}$ and remarkable capacity retention of near $90 \%$ at high sulfur loadings up to $8 \mathrm{mg} \mathrm{cm}^{-2}$. We 
believe that these findings open a new avenue toward the design of high energy rechargeable batteries through the exploration of metal-free catalyst materials.

\section{Methods}

Materials preparation. For BPQD preparation, BP crystal was purchased from Smart Elements. We added $45 \mathrm{mg}$ BP crystals into $20 \mathrm{~mL}$ NMP solution and ultrasonicated using an ultrasonic bath $(400 \mathrm{~W})$ at a temperature of $20^{\circ} \mathrm{C}$ through the whole experiment for $8.0 \mathrm{~h}$. The obtained BP solution was purified by centrifugation, with rates of $1000-3000 \mathrm{rpm}$ for $30 \mathrm{~min}$, to remove large particles at bottom. Then the BP suspension was further sonicated using probe sonication. The as-obtained BP/NMP solution was centrifuged at 4000,8000 , and 12,000 rpm for $30 \mathrm{~min}$, and the precipitates were collected and designated as BP-4K, BP-8K, and $\mathrm{BPQD}$, respectively. The yield of BPQD produced in this work is discussed in Supplementary Note 1 . The as-obtained BP NMP solutions were stored in an Arfilled glove box to ensure their stability. Without mention, all below experiments related to BP flakes were conducted in the glove box.

For porous carbon nanofiber synthesis, $0.5 \mathrm{~g}$ polyacrylonitrile was dissolved in $20 \mathrm{ml} \mathrm{N}, N$-dimethylformamide solvent and magnetically stirred for $8 \mathrm{~h}$ at $80^{\circ} \mathrm{C}$. Then, $1.0 \mathrm{~g}$ iron (III) acethylacetonate was added to the above solution and stirred for another $8 \mathrm{~h}$. The polymer mixture was electrospun into nanofibers using an electrospiner at $18 \mathrm{kV}$ with a constant flow rate of $1 \mathrm{~mL} \mathrm{~h}^{-1}$. The neat fibers were stabilized in air for $3 \mathrm{~h}$ at $220^{\circ} \mathrm{C}$ and sequentially carbonized in $\mathrm{Ar}$ atmosphere for $1 \mathrm{~h}$ at $650^{\circ} \mathrm{C}$ at a ramp rate of $3{ }^{\circ} \mathrm{C} \mathrm{min}^{-1}$. The resultant carbon nanofibers containing $\mathrm{Fe}_{3} \mathrm{C}$ particles were soaked in fuming $\mathrm{HNO}_{3}$ for $10 \mathrm{~h}$ to create hollow graphitic carbon spheres via removing the $\mathrm{Fe}_{3} \mathrm{C}$ catalyst. To open the graphitic carbon walls, the as-obtained porous carbon nanofibers were mixed with $\mathrm{KOH}$ at a mass ratio of $1: 4$ and transferred to a tube furnace and heated at $750^{\circ} \mathrm{C}$ for $0.5 \mathrm{~h}$ under $\mathrm{Ar}$ gas flow. Then the resulting product was washed with flooded amount of $\mathrm{HCl}(0.1 \mathrm{M})$ and DI water subsequently, before drying in vacuum oven for $8 \mathrm{~h}$ at $100^{\circ} \mathrm{C}$.

For PCNF/S composite preparation, the PCNF was mixed with sulfur particles at a mass ratio of 25:75. The PCNF/S mixture was then placed in a tube furnace with $\mathrm{Ar}$ flow and heated at $155^{\circ} \mathrm{C}$ for $12 \mathrm{~h}$ to infiltrate molten sulfur within PCNF host. The PCNF/S/BPQD composites were prepared by adding about $3 \mathrm{wt} \% \mathrm{BPQD}$ in PCNF/S composite in $\mathrm{CS}_{2}$ solution. $\mathrm{CS}_{2}$ can dissolve sulfur particles thus the $\mathrm{BPQD}$ and sulfur would be simultaneously re-infiltrated into PCNF host by capillary force. It is worth noting that the morphology and stability of BPQD would not be affected by $\mathrm{CS}_{2}$ solvent (Supplementary Fig. 15).

Materials characterization. For structural characterization of BP, Raman spectra were obtained from a Horiba Jobin Yvon HR800 Raman microscopic system equipped with a $488 \mathrm{~nm}$ laser operating at $180 \mathrm{~mW}$. The spot size of Raman laser was controlled near $1 \mu \mathrm{m}$. A TEM (JEOL $2100 \mathrm{~F}$ ) working at $200 \mathrm{kV}$ was used to estimate the morphological information of BP flakes and BPQD.

For PCNF and PCNF/S/BPQD characterization, the morphologies were investigated using a SEM (JEOL 6700) and a TEM. The STEM image and elemental mapping of PCNF/S/BPQD were conducted on the JEOL 2100-TEM equipped with EDS detector. The surface area and pore size distribution of PCNF host were determined from $\mathrm{N}_{2}$ adsorption/desorption isotherms at $77 \mathrm{~K}$ using an automated adsorption apparatus (Micromeritics, ASAP 2020). The chemical compositions of sulfur/carbon composites were evaluated by TGA (Q5000) at a ramp rate of $5.0^{\circ} \mathrm{C}$ $\min ^{-1}$ in nitrogen atmosphere.

\section{Adsorption and catalytic studies of black phosphorus quantum dots. $\mathrm{Li}_{2} \mathrm{~S}_{8}$} solution was prepared by mixing sulfur particles and $\mathrm{Li}_{2} \mathrm{~S}$ with a molar ratio of 7:1 in tetraglyme solvent, followed by stirring at $50^{\circ} \mathrm{C}$ for $8 \mathrm{~h}$ in an Ar-filled glove box. $1 \mathrm{mg}$ of BP-4K, BP-8K, and PBQD powders were dispersed individually in $10 \mathrm{~mL}$ $\mathrm{Li}_{2} \mathrm{~S}_{8} /$ tetraglyme solution with a concentration of $5 \mathrm{mmol} \mathrm{L}^{-1}$ in sulfur. To observe the color change, the mixtures were kept standing for $12 \mathrm{~h}$. The supernatant and the BP precipitates of the mixtures were studied by UV-vis spectrophotometry and XPS, respectively. The precipitates were obtained by centrifugation.

The nucleation and growth of $\mathrm{Li}_{2} \mathrm{~S}$ from soluble polysulfides were studied by potentiostatic deposition of $\mathrm{Li}_{2} \mathrm{~S}_{8}$ tetraglyme solution $\left(0.2 \mathrm{~mol} \mathrm{~L}^{-1}\right.$ based on sulfur $)$ on CF-based current collectors. CF papers were punched into disks with a diameter of $14 \mathrm{~mm}$ and about $0.40 \mathrm{mg}$ of BP-4K, BP-8K, and BPQD powders were separately dispersed on CF papers using pure ethanol as solvent. $25 \mu \mathrm{Li}_{2} \mathrm{~S}_{8}$ was dropped onto the $\mathrm{CF} / \mathrm{BP}$ current collectors as cathode. Lithium foil was employed at the counter electrode, which was separated with cathode by Celgard 2400 membrane and dropped with $25 \mu \mathrm{L} 0.50 \mathrm{~mol} \mathrm{~L}^{-1}$ lithium bis(trifluoromethanesulfonyl)imide (LiTFSI) tetraglyme electrolyte on the Li metal side. The cells were galvanostatically discharged to $2.06 \mathrm{~V}$ at a constant current density of $0.112 \mathrm{~mA}$, and then kept potentiostatically at $2.05 \mathrm{~V}$ for $\mathrm{Li}_{2} \mathrm{~S}$ to nucleate and grow until the current dropped below $10^{-5} \mathrm{~A}$. It took about $60,000 \mathrm{~s}$ and the energy was integrated to evaluate the capacities from deposition of lithium sulfide on various surfaces according to Faraday's law.

The catalytic property of the BPQDs was further studied by $\mathrm{CV}$ test of symmetric cells. The CF/BPQD and CF symmetric cells were prepared by assembling identical electrodes with a Celgard 2400 membrane as separator, and
$25 \mu \mathrm{Li}_{2} \mathrm{~S}_{4}\left(0.2 \mathrm{~mol} \mathrm{~L}^{-1}\right)$ tetraglyme electrolyte was added. CV was performance at $\mathrm{CHI} 660 \mathrm{c}$ electrochemical workstation at a scan rate of $1 \mathrm{mV} \mathrm{s}^{-1}$ between -1.0 and $1.0 \mathrm{~V}$. LSV studies were conducted using $\mathrm{CF} / \mathrm{BPQD}$ or $\mathrm{CF}$ as working electrode, lithium foil as counter electrode and $0.2 \mathrm{~mol} \mathrm{~L}^{-1} \mathrm{Li}_{2} \mathrm{~S}_{4}$ as catholyte. The cells were tested at electrochemical workstation between 1.7 and $2.8 \mathrm{~V}$ at a scan rate of 0.1 $\mathrm{mV} \mathrm{s}^{-1}$.

In situ characterization. In situ TEM experiment was conducted on a JEOL 2100TEM equipped with a Nanofactory scanning tunneling microscope (STM) holder. A thin layer of $\mathrm{Li}$ metal was scratched on the tip of a sharp $\mathrm{Cu}$ rode as reference electrode, while PCNF/S/BPQD fibers were dispersed on the tip of another $\mathrm{Cu}$ wire as working electrode. During the transfer of the STM holder assembled with two electrodes in TEM chamber, Ar gas flow was used to protect Li metal from moisture and oxygen. A potential of $-2.0 \mathrm{~V}$ vs. $\mathrm{Li} / \mathrm{Li}_{2} \mathrm{O}$ was applied to working electrode to drive the lithiation reaction once a physical contact is confirmed between two electrodes. In situ TB experiment was conducted by sealing a PCNF/S/ $\mathrm{BPOD}$ or PCNF/S cathode and a Li metal anode in a glass bottle filled with about $12.0 \mathrm{~mL} 1.0 \mathrm{~mol} \mathrm{~L}^{-1}$ LiTFSI 1,3-dioxolane: 1,2-dimethoxy (DOL: DME, 1/1 v/v) electrolyte. The transparent batteries were galvanostatically discharged at $0.10 \mathrm{~mA}$ for $8 \mathrm{~h}$, when optical images were taken to show the color change of electrolyte. In situ EIS measurements were performed on a Bio-Logic VSP-300 analyzer. The cells were assembled using PCNF/S/BPQD or PCNF/S cathodes and Li metal anodes in an Ar-filled glove box, to be discussed below. After every 20 min galvanostatic discharge at $0.1 \mathrm{C}$, we held the cells for $15 \mathrm{~min}$ to reach equilibrium before performing the EIS measurement. The EIS was conducted at a perturbation amplitude of $5 \mathrm{mV}$ in the frequency between $10 \mathrm{mHz}$ and $100 \mathrm{kHz}$. The EIS spectra were simulated using Z-view software.

Cell assembly and electrochemical performance. To measure of the electrochemical performance, the cathodes were prepared by mixing PCNF/S/BPQD composite, carbon black, and polyvinylidene fluoride binder at a mass ratio of 8:1:1 using NMP solvent. The slurry mixture was cast on aluminum foil and cut into discs $(\phi=14 \mathrm{~mm})$, giving rise to an average sulfur loading of about $2 \mathrm{mg} \mathrm{cm}^{-2}$. The cathodes were assembled into CR2032 coin cells using Li metal anode, $1 \mathrm{~mol} \mathrm{~L}^{-1}$ LiTFSI DOL/DME electrolyte with $1.0 \mathrm{wt} \% \mathrm{LiNO}_{3}$ additive, and polyethylene membrane (Celgard 2400) separator in an Ar-filled glove box. The electrolyte added in the cell is $80 \mu \mathrm{L}$, and the electrolyte/sulfur ratios are about 26 , $13,8.7$, and $6.5 \mathrm{~mL} \mathrm{~g}^{-1}$ for the electrodes with sulfur loadings of $2,4,6$, and $8 \mathrm{mg}$ $\mathrm{cm}^{-2}$, respectively. These values are comparable with previous reports ${ }^{18,19,46}$. The PCNF/BPQD/catholyte electrodes were prepared by dropping $45 \mu \mathrm{L} 1.5 \mathrm{~mol} \mathrm{~L}^{-1}$ $\mathrm{Li}_{2} \mathrm{~S}_{6}$ catholyte into PCNF/BPQD electrode substrate $\left(1 \times 1 \mathrm{~cm}^{2}\right.$, about $3 \mathrm{mg} \mathrm{cm}^{-2}$ with $2 \mathrm{wt} \%$ of BPQD). The polysulfide catholyte was prepared by mixing stoichiometric amount of sulfur and $\mathrm{Li}_{2} \mathrm{~S}$ in DOL/DME $=1 / 1 \mathrm{v} / \mathrm{v}$ with $1.85 \mathrm{~mol} \mathrm{~L}^{-1}$ LiTFSI and $0.2 \mathrm{~mol} \mathrm{~L}^{-1} \mathrm{LiNO}_{3}$, according to the previous work ${ }^{57}$. The final electrode achieved a high sulfur loading of $13.2 \mathrm{mg} \mathrm{cm}^{-2}$ and a sulfur content of $81 \mathrm{wt}$ $\%$. Additional blank electrolyte was used to wet separator to achieve an E/S ratio of $\sim 4 \mathrm{~mL} \mathrm{~g}^{-1}$. The cells were tested at different current densities between 1.7 and 2.8 $\mathrm{V}$ vs. $\mathrm{Li}^{+} / \mathrm{Li}$ on a LAND $2100 \mathrm{CT}$ battery tester. The post-mortem analysis was carried out by dissembling the cycled PCNF/S/BPQD electrodes in a glove box and washing with flooded amounts of DME to remove the salt and byproducts. Cycled electrodes were sealed in Ar-filled bottles before sending to SEM and TEM characterizations.

Theoretical calculations. DFT calculations were performed with the Vienna Ab initio Simulation Package (VASP) ${ }^{63,64}$, within the generalized gradient approximation proposed by Perdew, Burke, and Ernzerhof $(\mathrm{PBE})^{65}$. We used Grimme's DFT-D2 method ${ }^{66}$ to correct for the vdW interaction poorly described by standard DFT. We studied the energetics of adsorbed LiPSs on two types of BP structures: 2D-free-standing monolayer and one-dimensional bilayers zig-zag terminated BP nanoribbons, as represented in Supplementary Fig. 16. It has been shown that ZZ terminations are lower in energy than their arm-chair counterpart ${ }^{67}$. Therefore, we assume that all the BPQD edges are ZZ-terminated. DFT simulations were performed with a kinetic energy cutoff of $500 \mathrm{eV}$ and we used two $k$-points in the periodic directions to evaluate the integrals in the reciprocal space. Convergence was achieved when energy, force and stress reached a minimum of $5 \times 10^{-4} \mathrm{eV}$, $5 \times 10^{-2} \mathrm{eV} \AA^{-1}$, and $5 \times 10^{-2} \mathrm{GPa}$, respectively. The potential energy surface corresponding to the adsorption of LiPS at the terrace or at the edge of BP is rough and presents multiple minima. Therefore, to find the lowest energy location of adsorbed LiPS on BP, we performed for each compound, ab initio molecular dynamics simulations at $300 \mathrm{~K}$ with a kinetic energy cutoff of $300 \mathrm{eV}$ and a time step of $1.5 \mathrm{fs}$. Finally, we computed the binding energy $E_{\mathrm{b}}$ as:

$$
E_{\mathrm{b}}=E_{\mathrm{LiPS}}+E_{\mathrm{BP}}-E_{\mathrm{LiPS} @ \mathrm{BP}}
$$

with $E_{\mathrm{Lips}}, E_{\mathrm{BP}}$, and $E_{\mathrm{LiPS} @ \mathrm{BP}}$ the energy of LiPSs, BP, and LiPSs adsorbed on the terrace site (or at the edge site) of BP, respectively. Following this definition, higher binding energy implies more favorable adsorption. 


\section{Data availability}

The data that support the findings in this study are in the paper and/or the 524 Supplementary Information. Additional data are available from the authors upon reasonable 525 request.

Received: 26 February 2018 Accepted: 14 September 2018

Published online: 09 October 2018

\section{References}

1. Bruce, P. G., Freunberger, S. A., Hardwick, L. J. \& Tarascon, J. M. Li-O $\mathrm{O}_{2}$ and Li-S batteries with high energy storage. Nat. Mater. 11, 19-29 (2011).

2. Xu, Z. L., Liu, X., Luo, Y., Zhou, L. \& Kim, J. K. Nanosilicon anodes for high performance rechargeable batteries. Prog. Mater. Sci. 90, 1-44 (2017)

3. Manthiram, A., Fu, Y. \& Su, Y. Challenges and prospects of lithium-sulfur batteries. Acc. Chem. Res. 46, 1125-1134 (2013).

4. Wild, M. et al. Lithium sulfur batteries, a mechanistic review. Energy Environ. Sci. 8, 3477-3494 (2015).

5. Pang, Q., Liang, X., Kwok, C. Y. \& Nazar, L. F. Advances in lithium-sulfur batteries based on multifunctional cathodes and electrolytes. Nat. Energy 1, 16132 (2016)

6. Ji, X., Lee, K. T. \& Nazar, L. F. A highly ordered nanostructured carbon-sulphur cathode for lithium-sulphur batteries. Nat. Mater. 8, 500-506 (2009).

7. Su, Y. S. \& Manthiram, A. Lithium-sulphur batteries with a microporous carbon paper as a bifunctional interlayer. Nat. Commun. 3, 1166 (2012).

8. Xin, S. et al. Smaller sulfur molecules promise better lithium-sulfur batteries. J. Am. Chem. Soc. 134, 18510-18513 (2012).

9. Chong, W. G. et al. Lithium-sulfur battery cable made from ultralight, flexible graphene/carbon nanotube/sulfur composite fibers. Adv. Funct. Mater. 27, 1604815 (2017).

10. Xu, Z. L., Kim, J. K. \& Kang, K. Carbon nanomaterials for advanced lithium sulfur batteries. Nano Today 19, 84-107 (2018).

11. Pang, Q., Liang, X., Kwok, C. Y. \& Nazar, L. F. Review-the importance of chemical interactions between sulfur host materials and lithium polysulfides for advanced lithium-sulfur batteries. J. Electrochem. Soc. 162, A2567-A2576 (2015).

12. Li, L. et al. Stabilizing sulfur cathodes using nitrogen-doped graphene as a chemical immobilizer for Li-S batteries. Carbon N. Y. 108, 120-126 (2016).

13. Gao, J. et al. Vertically oriented arrays of $\mathrm{ReS}_{2}$ nanosheets for electrochemical energy storage and electrocatalysis. Nano Lett. 16, 3780-3787 (2016).

14. Hou, T. Z. et al. Lithium bond chemistry in lithium-sulfur batteries. Angew. Chem. Int. Ed. 56, 8178-8182 (2017).

15. Zheng, J. et al. Lewis acid-base interactions between polysulfides and metal organic framework in lithium sulfur batteries. Nano Lett. 14, 2345-2352 (2014).

16. Tao, X. et al. Balancing surface adsorption and diffusion of lithiumpolysulfides on nonconductive oxides for lithium-sulfur battery design. Nat. Commun. 7, 11203 (2016).

17. Liu, D. et al. Catalytic effects in lithium-sulfur batteries: promoted sulfur transformation and reduced shuttle effect. Adv. Sci. 5, 1700270 (2018).

18. Zhou, T. et al. Twinborn $\mathrm{TiO}_{2}-\mathrm{TiN}$ heterostructures enabling smooth trapping-diffusion-conversion of polysulfides towards ultralong life lithiumsulfur batteries. Energy Environ. Sci. 10, 1694-1703 (2017).

19. Yuan, Z. et al. Powering lithium-sulfur battery performance by propelling polysulfide redox at sulfiphilic hosts. Nano Lett. 16, 519-527 (2016).

20. Lei, T. et al. Multi-functional layered $\mathrm{WS}_{2}$ nanosheets for enhancing the performance of lithium-sulfur batteries. Adv. Energy Mater. 7, 1601843 (2017).

21. Keyes, R. W. The electrical properties of black phosphorus. Phys. Rev. 92 580-584 (1953).

22. Liu, H., Du, Y., Deng, Y. \& Ye, P. D. Semiconducting black phosphorus: synthesis, transport properties and electronic applications. Chem. Soc. Rev. 44, 2732-2743 (2015).

23. Liu, S. et al. Black phosphorus quantum dots used for boosting light harvesting in organic photovoltaics. Angew. Chem. Int. Ed. 56, 13717-13721 (2017).

24. Li, L. et al. Black phosphorus field-effect transistors. Nat. Nanotechnol. 9 372-377 (2014).

25. Li, W., Yang, Y., Zhang, G. \& Zhang, Y. W. Ultrafast and directional diffusion of lithium in phosphorene for high-performance lithium-ion battery. Nano Lett. 15, 1691-1697 (2015).

26. Sun, J. et al. Entrapment of polysulfides by a black-phosphorus-modified separator for lithium-sulfur batteries. Adv. Mater. 28, 9797-9803 (2016).
27. Li, L. et al. Phosphorene as a polysulfide immobilizer and catalyst in highperformance lithium-sulfur batteries. Adv. Mater. 29, 1602734 (2017).

28. Zhang, S., Ding, M. S., Xu, K., Allen, J. \& Jow, T. R. Understanding solid electrolyte interface film formation on graphite electrodes. Electrochem. SolidState Lett. 4, A206 (2001).

29. Wang, H. et al. High electrochemical selectivity of edge versus terrace sites in two-dimensional layered $\mathrm{MoS}_{2}$ materials. Nano Lett. 14, 7138-7144 (2014).

30. Babu, G., Masurkar, N., Al Salem, H. \& Arava, L. M. R. Transition metal dichalcogenide atomic layers for lithium polysulfides electrocatalysis. J. Am. Chem. Soc. 139, 171-178 (2017).

31. Hinnemann, B. et al. Biomimetic hydrogen evolution: $\mathrm{MoS}_{2}$ nanoparticles as catalyst for hydrogen evolution. J. Am. Chem. Soc. 127, 5308-5309 (2005).

32. Zhang, X. et al. Black phosphorus quantum dots. Angew. Chem. Int. Ed. 54, 3653-3657 (2015)

33. Sun, Z. et al. Ultrasmall black phosphorus quantum dots: synthesis and use as photothermal agents. Angew. Chem. Int. Ed. 54, 11526-11530 (2015).

34. Sun, J. et al. A phosphorene-graphene hybrid material as a high-capacity anode for sodium-ion batteries. Nat. Nanotechnol. 10, 980-985 (2015).

35. Xie, J. et al. A supramolecular capsule for reversible polysulfide storage/ delivery in lithium-sulfur batteries. Angew. Chem. Int. Ed. 56, 16223-16227 (2017).

36. Peng, H. J. et al. Enhanced electrochemical kinetics on conductive polar mediators for lithium-sulfur batteries. Angew. Chem. Int. Ed. 55, 12990-12995 (2016).

37. Fan, F. Y., Carter, W. C. \& Chiang, Y. M. Mechanism and kinetics of $\mathrm{Li}_{2} \mathrm{~S}$ precipitation in lithium-sulfur batteries. Adv. Mater. 27, 5203-5209 (2015).

38. Gerber, L. C. H. et al. Three-dimensional growth of $\mathrm{Li}_{2} \mathrm{~S}$ in lithium-sulfur batteries promoted by a redox mediator. Nano Lett. 16, 549-554 (2016).

39. Al Salem, H., Babu, G., V. Rao, C. \& Arava, L. M. R. Electrocatalytic polysulfide traps for controlling redox shuttle process of Li-S batteries. J. Am. Chem. Soc. 137, 11542-11545 (2015).

40. Babu, G., Ababtain, K., Ng, K. Y. S. \& Arava, L. M. R. Electrocatalysis of lithium polysulfides: current collectors as electrodes in Li/S battery configuration. Sci. Rep. 5, 8763 (2015).

41. Henkelman, G., Arnaldsson, A. \& Jónsson, H. A fast and robust algorithm for Bader decomposition of charge density. Comput. Mater. Sci. 36, 354-360 (2006).

42. Zhao, J., Yang, Y., Katiyar, R. S. \& Chen, Z. Phosphorene as a promising anchoring material for lithium-sulfur batteries: a computational study. $J$. Mater. Chem. A 4, 6124-6130 (2016).

43. Jiang, Z. T., Liang, F. X. \& Zhang, X. D. A comparative study on the edge states in phosphorene quantum dots and rings. Phys. Lett. A 381, 373-378 (2017).

44. $\mathrm{Xu}, \mathrm{Z}$. L. et al. In situ TEM study of volume expansion in porous carbon nanofiber/sulfur cathodes with exceptional high-rate performance. $A d v$. Energy Mater. 7, 1602078 (2017).

45. Huang, J. Q. et al. Novel interlayer made from $\mathrm{Fe}_{3} \mathrm{C} /$ carbon nanofiber webs for high performance lithium-sulfur batteries. J. Power Sources 285, 43-50 (2015)

46. Yin, L. C. et al. Understanding the interactions between lithium polysulfides and $\mathrm{N}$-doped graphene using density functional theory calculations. Nano Energy 25, 203-210 (2016).

47. Zhou, W. et al. Tailoring pore size of nitrogen-doped hollow carbon nanospheres for confining sulfur in lithium-sulfur batteries. Adv. Energy Mater. 5, 1401752 (2015).

48. Kim, H. et al. In situ TEM observation of electrochemical lithiation of sulfur confined within inner cylindrical pores of carbon nanotubes. Adv. Energy Mater. 5, 1501306 (2015)

49. Liu, X. H. et al. Ultrafast electrochemical lithiation of individual Si nanowire anodes. Nano. Lett. 11, 2251-2258 (2011)

50. Pan, H. et al. Non-encapsulation approach for high-performance Li-S batteries through controlled nucleation and growth. Nat. Energy 2, 813-820 (2017).

51. Tan, G. et al. Burning lithium in $\mathrm{CS}_{2}$ for high-performing compact $\mathrm{Li}_{2} \mathrm{~S}$-graphene nanocapsules for Li-S batteries. Nat. Energy 2, 17090 (2017).

52. Park, C. M. \& Sohn, H. J. Black phosphorus and its composite for lithium rechargeable batteries. Adv. Mater. 19, 2465-2468 (2007).

53. Aurbach, D. et al. On the surface chemical aspects of very high energy density, rechargeable Li-sulfur batteries. J. Electrochem. Soc. 156, A694 (2009).

54. Jozwiuk, A. et al. The critical role of lithium nitrate in the gas evolution of lithium-sulfur batteries. Energy Environ. Sci. 9, 2603-2608 (2016).

55. Li, Y. J., Fan, J. M., Zheng, M. S. \& Dong, Q. F. A novel synergistic composite with multi-functional effects for high-performance Li-S batteries. Energy Environ. Sci. 9, 1998-2004 (2016).

56. Sun, Z. et al. Conductive porous vanadium nitride/graphene composite as chemical anchor of polysulfides for lithium-sulfur batteries. Nat. Commun. 8 14627 (2017) 
57. Chung, S. H. \& Manthiram, A. Designing lithium-sulfur cells with practically necessary parameters. Joule 2, 710-724 (2018).

58. Pan, H. et al. Addressing passivation in lithium-sulfur battery under lean electrolyte condition. Adv. Funct. Mater. 8, 1707234 (2018).

59. Wang, H. et al. Tailored reaction route by micropore confinement for Li-S batteries operating under lean electrolyte conditions. Adv. Energy Mater. 8, 1800590 (2018).

60. Zheng, C. et al. Propelling polysulfides transformation for high-rate and longlife lithium-sulfur batteries. Nano Energy 33, 306-312 (2017)

61. Li, Z., Zhang, J. \& Lou, X. W. Hollow carbon nanofibers filled with $\mathrm{MnO}_{2}$ nanosheets as efficient sulfur hosts for lithium-sulfur batteries. Angew. Chem. Int. Ed. 54, 12886-12890 (2015).

62. Cui, Z., Zu, C., Zhou, W., Manthiram, A. \& Goodenough, J. B. Mesoporous titanium nitride-enabled highly stable lithium-sulfur batteries. Adv. Mater. 28, 6926-6931 (2016).

63. Kresse, G. \& Furthmüller, J. Efficiency of ab-initio total energy calculations for metals and semiconductors using a plane-wave basis set. Comput. Mater. Sci. 6, 15-50 (1996).

64. Kresse, G. \& Furthmüller, J. Efficient iterative schemes for $a b$ initio total-energy calculations using a plane-wave basis set. Phys. Rev. B 54, 11169-11186 (1996).

65. Perdew, J. P., Burke, K. \& Ernzerhof, M. Generalized gradient approximation made simple. Phys. Rev. Lett. 77, 3865-3868 (1996).

66. Grimme, S., Antony, J., Ehrlich, S. \& Krieg, H. A consistent and accurate ab initio parametrization of density functional dispersion correction (DFT-D) for the 94 elements H-Pu. J. Chem. Phys. 132, 154104 (2010).

67. Lee, Y. et al. Atomic-scale imaging of few-layer black phosphorus and its reconstructed edge. J. Phys. D Appl. Phys. 50, 84003 (2017).

\section{Acknowledgements}

This work was supported by the Research Grants Council (RGC) of Hong Kong (Project Nos. PolyU 153271/16 P and PolyU 153039/17P), the Hong Kong Polytechnic University (Project No.:1-ZVGH), Korea Research Fellowship Program through the National Research Foundation of Korea (NRF) funded by the Ministry of Science and ICT (KRF Project No. 2017H1D3A1A01013931). Q.Z. thanks the support of National Key Research and Development Program (Project Nos. 2016YFA0202500 and 2015CB932500). Z.L.X. and K.K. are grateful for the financial support from Institute of Basic Science (IBS) at SNU. Z.L.X. thanks Dr. Sung Joo Kim at SNU for TEM assistance.

\section{Author contributions}

S.P.L. and Z.L.X. conceived the idea, Z.L.X. prepared the materials, conducted the electrochemical experiments, and characterization of materials, S.L. synthesized and characterized black phosphorous materials, N.O. conducted the DFT calculations. L.Z., F. S., W.L., and K.K. contributed to the characterizations and discussions of the results. S.P. L. and Q.Z. revised the manuscript written by Z.L.X. and all the authors commented on the manuscript.

\section{Additional information}

Supplementary Information accompanies this paper at https://doi.org/10.1038/s41467018-06629-9.

Competing interests: The authors declare no competing interests.

Reprints and permission information is available online at http://npg.nature.com/ reprintsandpermissions/

Publisher's note: Springer Nature remains neutral with regard to jurisdictional claims in published maps and institutional affiliations.

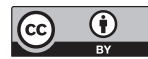

Open Access This article is licensed under a Creative Commons Attribution 4.0 International License, which permits use, sharing, adaptation, distribution and reproduction in any medium or format, as long as you give appropriate credit to the original author(s) and the source, provide a link to the Creative Commons license, and indicate if changes were made. The images or other third party material in this article are included in the article's Creative Commons license, unless indicated otherwise in a credit line to the material. If material is not included in the article's Creative Commons license and your intended use is not permitted by statutory regulation or exceeds the permitted use, you will need to obtain permission directly from the copyright holder. To view a copy of this license, visit http://creativecommons.org/ licenses/by/4.0/.

(C) The Author(s) 2018 